\title{
Der p2-A20 DNA vaccine attenuates allergic inflammation in mice with allergic rhinitis
}

\author{
WENHUI HU ${ }^{1,2^{*}}$, LI MA $^{1 *}$, GUI YANG ${ }^{1}$, XIANHAI ZENG ${ }^{1}$, JIANGQI LIU ${ }^{1}$, \\ BAOHUI CHENG ${ }^{1}$, TIANYONG HU ${ }^{1}$, HAILIANG ZHAO ${ }^{1}$ and ZHIQIANG LIU ${ }^{1}$ \\ ${ }^{1}$ Department of Otolaryngology, Longgang ENT Hospital and Shenzhen Key Laboratory of ENT, Institute of ENT, \\ Shenzhen, Guangdong 518172; ${ }^{2}$ Zunyi Medical College, Zunyi, Guizhou 519090, P.R. China
}

Received April 1, 2019; Accepted August 30, 2019

DOI: $10.3892 / \mathrm{mmr} .2019 .10760$

\begin{abstract}
Allergic rhinitis (AR) is a common disease that requires more convenient, safe and effective antigen-specific immunotherapies. The present study aimed to investigate the therapeutic effect of intranasal administration of a eukaryotic expression vector co-expressing Der p2 and A20 protein (pVAX1-Der p2-A20) on mice with allergic rhinitis. The pVAX1-Der p2-A20 vaccine was prepared and encapsulated into poly(L-lactide-co-glycolide) (PLGA) nanoparticles. An allergic rhinitis Balb/c mouse model was established through intraperitoneal sensitization with recombinant Der p2 and cholera toxin followed by intranasal challenge with recombinant Der p2. The treatment effect of the DNA vaccine on nasal allergic inflammation was evaluated, and serum IgE, sIgE, IgG and cytokine levels were determined by ELISA. The percentage of $\mathrm{CD} 4^{+} \mathrm{CD} 25^{+} \mathrm{Foxp} 3^{+}$Tregs in the spleen was detected by flow cytometry. The DNA vaccine co-expressing Der $\mathrm{p} 2$ and A20 was successfully constructed and encapsulated into PLGA nanoparticles. Der p2-A20 DNA vaccine intranasal administration markedly ameliorated Der p2-induced nasal allergic inflammation. The serum Der p2-specific IgE, IL-4 and IL-13 expression levels were inhibited, while the Der p2-specific IgG1, IgG2a and IFN- $\gamma$ expression levels in the serum and splenic $\mathrm{CD} 4^{+} \mathrm{CD} 25^{+} \mathrm{Foxp} 3^{+}$Treg population were significantly increased after Der p2-A20 DNA vaccine treatment. These results indicated that the Der p2-A20 DNA vaccine alleviates nasal allergic inflammation and promotes splenic Treg population in mice with allergic rhinitis.
\end{abstract}

Correspondence to: Professor Zhiqiang Liu or Professor Hailiang Zhao, Department of Otolaryngology, Longgang ENT Hospital and Shenzhen Key Laboratory of ENT, Institute of ENT, 3004 Longgang Avenue, Longgang, Shenzhen, Guangdong 518172, P.R. China

E-mail: liuzhiqiang05312438@126.com

E-mail: 825109331@qq.com

*Contributed equally

Key words: allergic rhinitis, Dermatophagoides pteronyssinus, DNA vaccine, $\mathrm{A} 20, \mathrm{NF}-\kappa \mathrm{B}$

\section{Introduction}

Allergic rhinitis (AR) is a non-infectious nasal mucosal disease caused by the exposure of susceptible individuals to exogenous allergens and the release of IgE-mediated histamine and other mediators, and it is accompanied by immune cell activation and cytokine secretion (1). As a common otolaryngology disease, the incidence of AR has increased greatly in the past decades (2). AR has become a global public health problem and caused huge economic burden to individuals, families and society. At present, the treatment of AR mainly includes avoidance of contact with susceptible allergens, anti-inflammation, antihistamine and other symptomatic treatments, which are far from satisfactory. Allergen-specific immunotherapy (AIT) is the only WHO approved aetiological treatment for allergic diseases that can effectively change the natural process of AR (3). At present, the clinically used desensitization agents are mainly from allergen crude extracts, which contain complex components, resulting in poor stability, difficulty in standardization, and serious side effects (4). Therefore, it is particularly important to develop new, safe and efficient methods for the prevention and treatment of AR.

The dust mite is an important allergen of AR, and studies have revealed that approximately $70-80 \%$ of patients with upper respiratory tract allergy have positive reactions to dust mites, among which Der f2 and Der p2 have the highest serum positive rate $(5,6)$. A20, also known as tumour necrosis factor-induced protein 3 (TNFAIP3), is a ubiquitin-modified enzyme protein encoded by the TNFAIP 3 gene in the cytoplasm that regulates a variety of immune cell functions and is involved in maintaining immune homeostasis (7). Mutations in the A20 gene are associated with inflammation, allergic disease and tumourigenesis (8). Mice with A20 gene deletion have severe inflammation and tissue damage, and A20 overexpression improves the tolerance of intestinal mucosa to LPS and enhances the intestinal mucosal barrier function. A20 plays an important role in the pathogenesis of allergic diseases, and its absence can lead to the occurrence of allergic asthma (8).

Studies have revealed that a DNA vaccine displays not only immunogenicity and safety but also greater flexibility than previous protein vaccines for its convenient modification and construction (9). Compared with the AIT and recombinant 
allergen vaccines, a DNA vaccine induces Th1 cell reaction, reduces allergen-specific IgE, and increases the concentration of $\operatorname{IgG} 2 \mathrm{a}$ by endogenous expression of allergens (10), thus becoming a promising strategy for AR-specific immune therapy. Further studies have indicated that the combination of an allergen gene with a regulatory molecule through genetic engineering technology improves the therapeutic effect of a DNA vaccine. The fusion of allergen ovalbumin with heat shock protein (Hsp65) has been revealed to attenuate an established Th2 upper respiratory tract allergic inflammation in mice (10), and a fusion DNA vaccine constructed by malaria antigen and chemokine MIP3 CCL20 has been revealed to effectively prevent the infection of malaria parasites (11). In the present study, a DNA vaccine co-expressing Der p2 and A20 was constructed and encapsulated into PLGA nanoparticles, and its specific immunotherapeutic effect on allergic inflammation in the Der p2-induced AR mouse model and potential mechanism were evaluated.

\section{Materials and methods}

Reagents. Biotin-labelled sheep anti-mouse IgE antibody (cat. no. 1110-08) was purchased from Southern Biotech. HRP-labelled sheep anti-mouse IgG1 antibody (cat. no. A10551) and HRP-labelled sheep anti-mouse IgG2a antibody (cat. no. A10685) was purchased from Life Technologies; Thermo Fisher Scientific, Inc. HRP-labelled streptavidin (cat. no. A0303) was obtained from Beyotime Institute of Biotechnology. Mouse IL-4, IL-10, IL-13, TNF- $\alpha$, IFN- $\gamma$ and TGF- $\beta$ ELISA Kits were purchased from R\&D Systems. Cholera toxin and poly (lactic-co-glycolic acid) were purchased from SigmaAldrich; Merck KGaA. APC rat anti-mouse CD4 antibody (cat. no. 553051), FITC rat anti-mouse CD25 antibody (cat. no. 558689) and PE rat anti-mouse Foxp3 (cat. no. 560408) were obtained from BD Pharmingen; BD Biosciences. Anti-A20 antibody (product code ab74037) was purchased from Abcam, and anti-p65 antibody (product no. 3033S) and anti- $\beta$-actin antibody (4967S) were obtained from Cell Signaling Technology. The 293T cell line (CBP60439) was obtained from Cobioer.

Construction of DNA vaccine. For construction of the DNA vaccine co-expressing Der p2 and A20 (pVAX1-Der p2-A20), the coding sequence of Der p2 (FM177223.1) and TNFAIP3 (GenBank: KJ892292.1) were synthesized by GeneCreate Biotech and inserted into the pVAX1 vector. The mCherry gene sequence, which is a fluorescent protein derived from the tetrameric Discosoma sp., was amplified by polymerase chain reaction (PCR) from the pmCherry-N1 vector (presented by Jinan University, China) and subcloned into pVAX1-Der p2-A20 to establish the pVAX1-mCherry-Der p2-A20 expression vector. The recombinant pVAX1-Der p2-A20 and pVAX1-mCherry-Der p2-A20 expression vectors were encapsulated into poly(L-lactide-co-glycolide) (PLGA) (Sigma-Aldrich; Merck KGaA) to form nanoparticles via the emulsion method before intranasal administration (Fig. 1). In addition, pET-32a-Der p2 was also constructed for recombinant Der $\mathrm{p} 2$ expression and purification $(>95 \%)$. The coding sequence of the Der 2 from Genebank was synthesized and inserted into pET-32a vector (Thermo Fisher Scientific,
Inc.). Then the recombinant vector was transfected into BL21 Escherichia coli for Der p2 expression, purification and identification (data not shown). For evaluation of the transfection effect of constructed DNA vaccine in vitro, the 293T cell line was cultured and transfected in DMEM (product no. 10-013-CVRC; Corning Incorporated) supplemented with $10 \%$ fetal bovine serum (Sigma-Aldrich; Merck $\mathrm{KGaA}$ ) and penicillin-streptomycin (Corning Incorporated) in a $125 \mathrm{ml}$ polycarbonate flask (Corning Incorporated) under the following conditions: $37^{\circ} \mathrm{C}, 5 \% \mathrm{CO}_{2}$ and $80 \%$ humidity.

Mice. In total, 30 female BALB/c mice (6-8 weeks old) were purchased from Guangzhou Experimental Animal Centre and were housed under pathogen-free conditions. The experimental procedures were conducted in accordance with the approval and guidelines of the Institutional Animal Care and Use Committee of the ENT Institute of Shenzhen (no. 201702013). Animal suffering and the number of animals used in this study were minimized. Mice were acclimated for 7 days before experiments and were monitored daily for signs of distress and advanced nasal inflammation during experiments.

Induction of nasal allergic inflammation in mice. The allergic rhinitis (AR) murine model was performed according to other and our previous procedures (12-14). Mice were sensitized with recombinant Der p2 $(10 \mu \mathrm{g})$ and cholera toxin (CT, $1 \mu \mathrm{g} /$ mouse) diluted in sterile normal saline $(0.1 \mathrm{ml} /$ mouse $)$ three times by intraperitoneal injection (ip) on days 0,7 and 14 . The sensitized mice in different groups ( $\mathrm{n}=6$ per group) were intranasally treated as follows ( $20 \mu \mathrm{l} /$ mouse) every 3 days for a total of 5 treatments from day 21: Sterile normal saline, AR group; pVAX1-Der p2-A20 nanoparticles $(100 \mu \mathrm{g})$, PpDA group; blank PLGA treatment $(100 \mu \mathrm{g})$, PLGA group; and naked pVAX1-Der p2-A20 (100 $\mu \mathrm{g}$; no encapsulation), pDA group. Mice were intranasally challenged three times daily with recombinant Der p2 (10 $\mu \mathrm{g} ; 20 \mu \mathrm{l}$ of $0.5 \mathrm{mg} / \mathrm{ml}$ Der p2) after the final treatment. Control groups (Con) were treated with normal saline by ip and nasal challenge. The frequencies of nose scratching and sneezing in each group were recorded within $30 \mathrm{~min}$ after the last challenge. Twenty-four hours after the last challenge, mice were sacrificed by cervical dislocation under $4 \%$ isoflurane (Youcheng Biotech) anesthesia. Samples were collected from each mouse for further evaluation (Fig. 2).

Symptom scores. The frequencies of nose scratching and sneezing in each group were counted within 30 min after the last intranasal challenge by recombinant Der $\mathrm{p} 2$ to evaluate the symptom scores according to a reported procedure (15).

Histology. The nasal mucosa was collected and fixed in $4 \%$ paraformaldehyde overnight and processed for paraffin embedding. Sections ( $4 \mu \mathrm{m}$ thick) were prepared and stained with haematoxylin and eosin (H\&E). Images were obtained using a microscope (Nikon Corporation; magnification of $\mathrm{x} 200$ ) for detection of inflammatory cell infiltration.

Enzyme-linked immunosorbent assay (ELISA). Mouse sera from each group were collected after the last challenge. 
A

$\begin{array}{ccc}100 & 110 & 120 \\ \text { TTGGTCGCAGCCGT TGCTCGTGATCAAGTCGATGTCAAAGATTGTGCC }\end{array}$

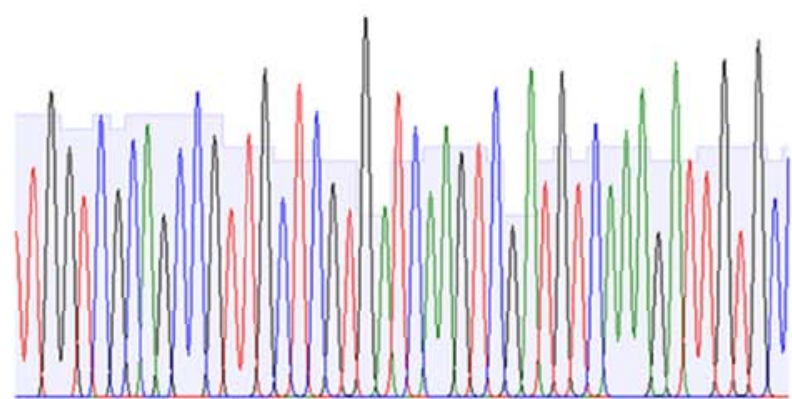

B

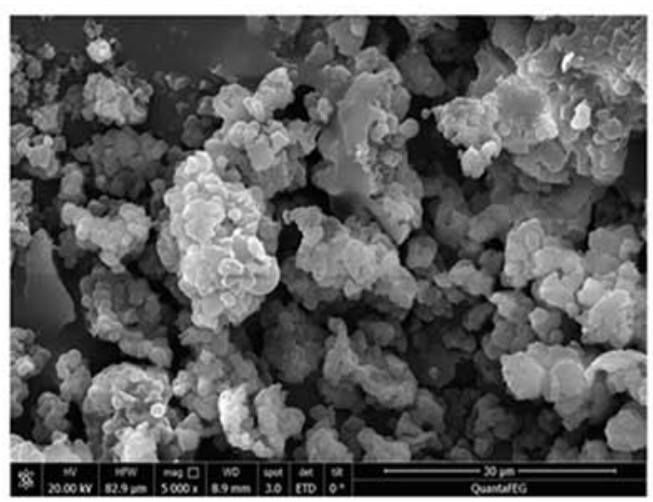

C
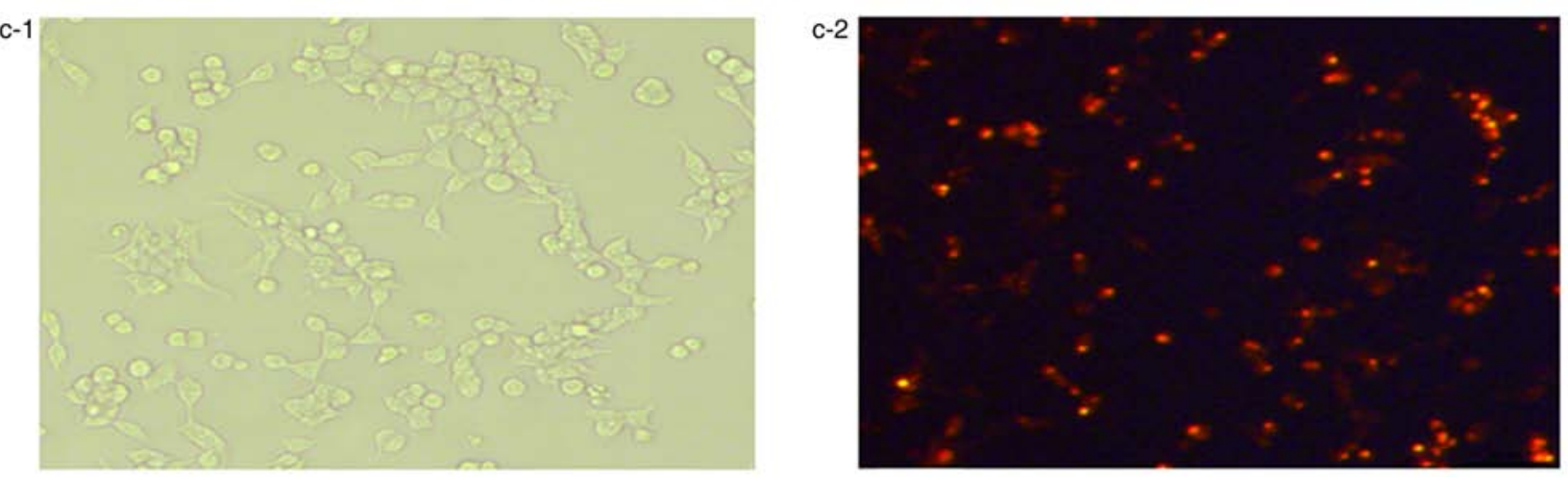

Figure 1. pVAX1-Der p2-A20 expression vector construction and transfection. (A) Recombinant pVAX1-Der p2-A20 partial sequencing results. (B) Scanning electron microscopy image of PLGA-encapsulated pVAX1-Der p2-A20 nanoparticles (magnification, x20,000). (C) The 293T cell line was transfected with PLGA-pVAX1-mCherry-Der p2-A20 nanoparticles (c1, 293T cell lines; and c2, confocal microscope image showing 293T cells transfected with pVAX1-mCherry-Der p2-A20 nanoparticles (magnification, x200). mCherry, red fluorescent protein; PLGA, poly(L-lactide-co-glycolide).

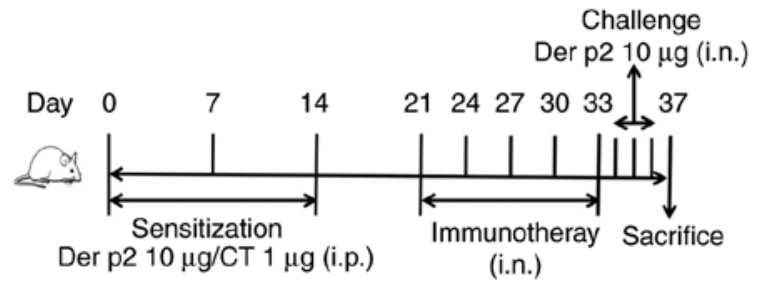

Figure 2. Schematic outline of allergic rhinitis induction and treatment. $\mathrm{BALB} / \mathrm{c}$ mice were sensitized with recombinant Der $\mathrm{p} 2$ and $\mathrm{CT}$ three times by ip on days 0,7 and 14 . The sensitized mice in different groups $(n=6$ per group) were then intranasally treated every 3 days for a total of 5 treatments from day 21 as follows: Sterile normal saline in the AR group, pVAX1-Der p2-A20 nanoparticles in the PpDA group (100 $\mu$ g, pVAX1-Der p2-A20), blank PLGA treatment in the PLGA group (100 $\mu \mathrm{g}$, PLGA) and naked pVAX1-Der p2-A20 treatment in the pDA group (100 $\mu \mathrm{g}$, pVAX1-Der p2-A20 without being encapsulated into PLGA). Mice were intranasally challenged three times daily with recombinant Der p2 $(10 \mu \mathrm{g})$ after the final treatment. The Con group was treated with normal saline by ip and nasal challenge. CT, cholera toxin; ip, intraperitoneal injection; Con, control; AR, allergic rhinitis; PLGA, poly(L-lactide-co-glycolide).

Serum concentrations of Der p2-specific antibodies including IgE (cat. no. 1110-08), IgG1(cat. no. A10551) from Southern Biotech, IgG2a (cat. no. A10685; Life Technologies; Thermo Fisher Scientific, Inc.), and cytokines including IL-4 (cat. no. M4000B), IL-10 (cat. no. M1000B), IL-13 (cat. no. M1300CB), IFN- $\gamma$ (cat. no. MIF00) and TGF- $\beta 1$ (cat. no. MB100B) were detected using ELISA kits (R\&D Systems) following the manufacturers' instructions.
Flow cytometry. Splenic mononuclear cells were collected from culture, fixed with $1 \%$ formaldehyde and permeabilization buffer $(0.1 \%$ Triton $\mathrm{X}-100)$ for $30 \mathrm{~min}$ at $4^{\circ} \mathrm{C}$, washed three times with $1 \%$ bovine serum albumin (BSA)/PBS and blocked for $30 \mathrm{~min}$ at $4^{\circ} \mathrm{C}$ with $1 \% \mathrm{BSA}$. Cells were incubated with APC-conjugated rat anti-mouse CD4, FITC-conjugated rat anti-mouse CD25 and PE-conjugated rat anti-mouse Foxp3 antibodies or isotype $\mathrm{IgG}$ for $1 \mathrm{~h}$ at room temperature. After washing with PBS, cells were analysed with a flow cytometer (FACSCanto II; BD Biosciences) and FlowJo V10 software (FlowJo, LLC).

Statistical analysis. SPSS 13.0 software (SPSS, Inc.) was used for statistical analysis. All values are presented as the mean \pm standard deviation (SD) of a minimum of three independent experiments. Data were analysed using one-way analysis of variance followed by Tukey's post hoc test for multiple comparisons. $\mathrm{P}<0.05$ was considered to indicate a statistically significant difference.

\section{Results}

Der $p 2$ and A20 DNA vaccine construction and in vitro transfection identification. The coding sequences of Der p2 [437 base pairs (bp)] and A20 (2,373 bp) were cloned into pVAX1 (3,000 bp). The constructed recombinant pVAX1-Der p2-A20 vector was confirmed by plasmid sequencing (Fig. 1A). The pVAX1-Der p2-A20 vector was then encapsulated into poly L-lactide-co-glycolide (PLGA) after construction to form 
a nanoparticle (Fig. 1B). To evaluate the transfection effect of the DNA vaccine, a pVAX1-mCherry-Der p2-A20 vector was also constructed by subcloning the mCherry-coding gene into pVAX1-Der p2-A20. The transfection effect of pVAX1-mCherry-Der p2-A20 in vitro and the expression of Der p2-A20-mCherry fusion protein in the 293T cell line were evaluated. Obvious red fluorescence was observed in the $293 \mathrm{~T}$ cells after transfection with pVAX1-mCherry-Der p2-A20 for $48 \mathrm{~h}$ (Fig. 1C). This result indicated that pVAX1-Der p2-A20 was successfully constructed and efficiently transfected into $293 \mathrm{~T}$ cells for fusion protein expression.

Der p2-A20 DNA vaccine inhibits Der p2-induced nasal allergic inflammation. A mouse model with AR was established, and the mice were treated with the Der p2-A20 DNA vaccine (Fig. 2). The results revealed that mice in the AR group had more denuded skin around the nose, increased scratching and sneezing frequencies (Fig. 3B and C), mononuclear cell infiltration in the nasal mucosa (Fig. 3A and D), and increased serum Der $\mathrm{p} 2$ specific IgE, IL-4 and IL-13 levels (Fig. 4) compared with mice in the control group. Der p2-A20 DNA vaccine administration in the PpDA group significantly reduced the scratching and sneezing events compared with administration in the AR and PLGA treatment groups (Fig. 3B and C). Histopathologic analysis of the AR group revealed evident nasal mucosal inflammation and increased mononuclear cells, and the Der p2-A20 DNA vaccine significantly inhibited the allergic inflammation compared with analysis of the AR and PLGA groups (Fig. 3A and D). Der p2-A20 DNA vaccine treatment in the PpDA group decreased scratching events, decreased sneezing events and inhibited nasal inflammation more efficiently than treatment with the naked plasmid DNA in the pDA group. These results indicated that the mouse model with AR was established successfully, and the Der p2-A20 DNA vaccine inhibits Der p2-induced nasal allergic inflammation.

Der p2-A20 DNA vaccine modulates the serum levels of cytokines and antibodies involved in nasal allergic inflammation. The serum levels of IL-4, IL-13, and TNF- $\alpha$ were increased while the levels of IL-10, IFN- $\gamma$ and TGF- $\beta$ were decreased in the AR group compared with those in the control group after the Der p2 challenge (Fig. 4). Intranasal treatment with the Der p2-A20 DNA vaccine suppressed IL-4, IL-13 and TNF- $\alpha$ secretion but improved the levels of IL-10, IFN- $\gamma$ and TGF- $\beta$ expression in the serum compared with the AR group (Fig. 4). Moreover, serum Der p2-specific IgE (sIgE), sIgG1 and sIgG2a antibodies were also evaluated. sIgE was significantlyy increased, whereas sIgG1 and sIgG2a levels were increased slightly in the AR group compared with those in the control group. The DNA vaccine enhanced sIgG1 and sIgG2a expression but decreased sIgE levels compared with the AR or pDA group (Fig. 4). These results indicated that the Der p2-A20 DNA vaccine may regulate Th1/Th2 cytokine expression and increase IL-10, TGF- $\beta$, sIgG1 and sIgG2a neutralizing antibody levels.

Der p2-A20 DNA vaccine increases splenic Treg population. To elucidate whether the inhibitory effects of the Der p2-A20 DNA vaccine on nasal inflammation in mice with allergic rhinitis were related to the induction of Tregs, the Treg population in the splenic $\mathrm{CD} 4^{+} \mathrm{T}$ cells was further analysed by flow cytometry (Fig. 5A). The results revealed that the frequency of $\mathrm{CD} 4{ }^{+} \mathrm{CD} 25^{+} \mathrm{Foxp}^{+} \mathrm{T}$ cells in splenic mononuclear cells was decreased in the AR group (2.64\%) compared with that in the control group $(4.55 \%)$, while this frequency was increased in the PpDA group (7.49\%) after DNA vaccine treatment (Fig. 5B and C). These results indicated that the Der p2-A20 DNA vaccine promotes Treg proliferation in mice with allergic rhinitis.

\section{Discussion}

As a common and frequently occurring disease in clinical practice, AR is a type of IgE-mediated non-infectious inflammation characterized by Th2 cell polarization (16). Currently, the symptomatic treatment based on histamine and other anti-inflammatory drugs cannot achieve a satisfactory therapeutic effect. The present study revealed that intranasal administration of the Der p2-A20 DNA vaccine markedly ameliorated Der p2-induced nasal mucosal proinflammatory cell infiltration. Serum Der p2-specific IgE, IL-4 and IL-13 expression levels were decreased, while serum Der p2-specific IgG1, IgG2a, IFN- $\gamma$, IL-10 and TGF- $\beta$ expression levels as well as the splenic $\mathrm{CD} 4^{+} \mathrm{CD} 25^{+} \mathrm{Foxp} 3^{+}$Treg population were significantly increased after Der p2-A20 DNA vaccine treatment. These results indicated that the Der p2-A20 DNA vaccine alleviates nasal allergic inflammation in mice with allergic rhinitis and promotes splenic Treg population. Therefore, DNA vaccine is a promising new direction for prevention and treatment of various pathogens. Compared with traditional protein-based vaccines, DNA vaccines are more stable, inexpensive, and can be conveniently produced. Biomaterial-based delivery systems that encapsulate plasmid DNA represent a promising strategy that can increase DNA vaccine internalization, transfection efficiency and mucosal uptake (10). Genetic immunization with a non-viral vector DNA vaccine has been employed in a broad range of therapeutic applications, including infectious diseases, cancers and other diseases $(17,18)$. Treatment strategies using plasmid DNA vaccine-encoding allergens in allergic diseases have been revealed to induce Th1 cell response, IFN- $\gamma$ production and $\mathrm{IgG} 2 \mathrm{a}$ production as well as to reduce the desensitization-induced $\operatorname{IgE}$ production and anaphylactic reactions compared with crude extracts used in allergen-specific immunotherapy $(19,20)$. CryJ-LAMP DNA vaccines co-expressing CryJ1 or CryJ2 from Japanese red cedar combined with lysosomal-associated membrane protein-1 (LAMP-1) were revealed to induce robust Th1-type immune responses as well as IgG2a and IgG1 antibody secretion in an allergic murine model (21). DNA-encoding Der p2 prevented the development of house dust mite-induced respiratory allergies by enhancing the Th1 cell immune response, reducing allergen-specific IgE, reducing IL-5, inducing IgG2a and inducing IFN- $\gamma(22)$. As a negative regulator of $\mathrm{NF}-\kappa \mathrm{B}$, A20 not only inhibited the activity of NF- $\kappa \mathrm{B}$ but also further blocked the release of various inflammatory factors induced by $N F-\kappa B$ (23). Loss of A20 in lung epithelium abolished the protective effect of farm dust and endotoxin to allergy and asthma (8), and deficiency of A20 promoted antigen 
A

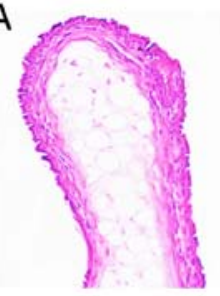

Con

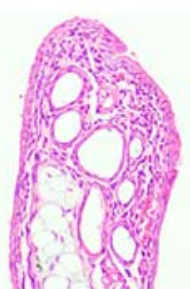

AR

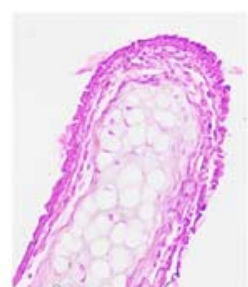

PpDA

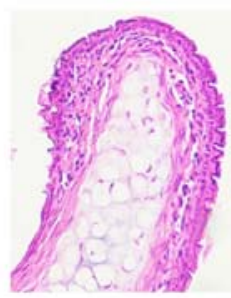

PLGA

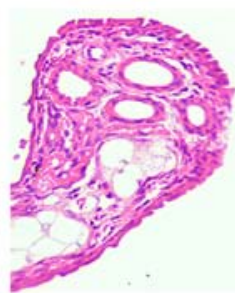

pDA
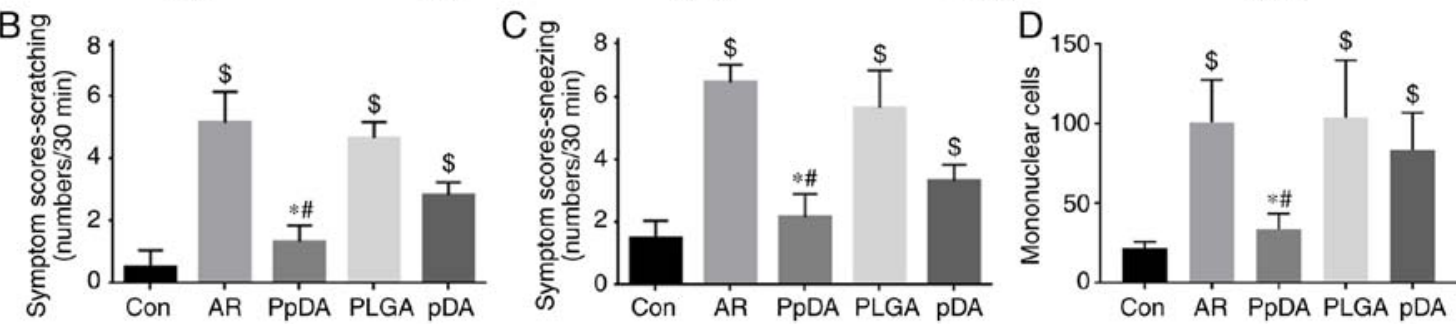

Figure 3. Der p2-A20 DNA vaccine ameliorates nasal allergic inflammation. (A) Representative H\&E staining (magnification x200) of nasal tissue sections from each group. Histogram represents the scores of (B) nose scratching and (C) sneezing. (D) Mononuclear cell infiltration in nasal mucosa. Data are obtained from three independent experiments and are presented as the mean $\pm \mathrm{SD}$. ${ }^{\$} \mathrm{P}<0.05$ vs. the Con group; ${ }^{*} \mathrm{P}<0.01$ vs. the $\mathrm{AR}$ group; ${ }^{\#} \mathrm{P}<0.05$ vs. the $\mathrm{pDA}$ group. Con, control; AR, allergic rhinitis; PLGA, poly(L-lactide-co-glycolide).
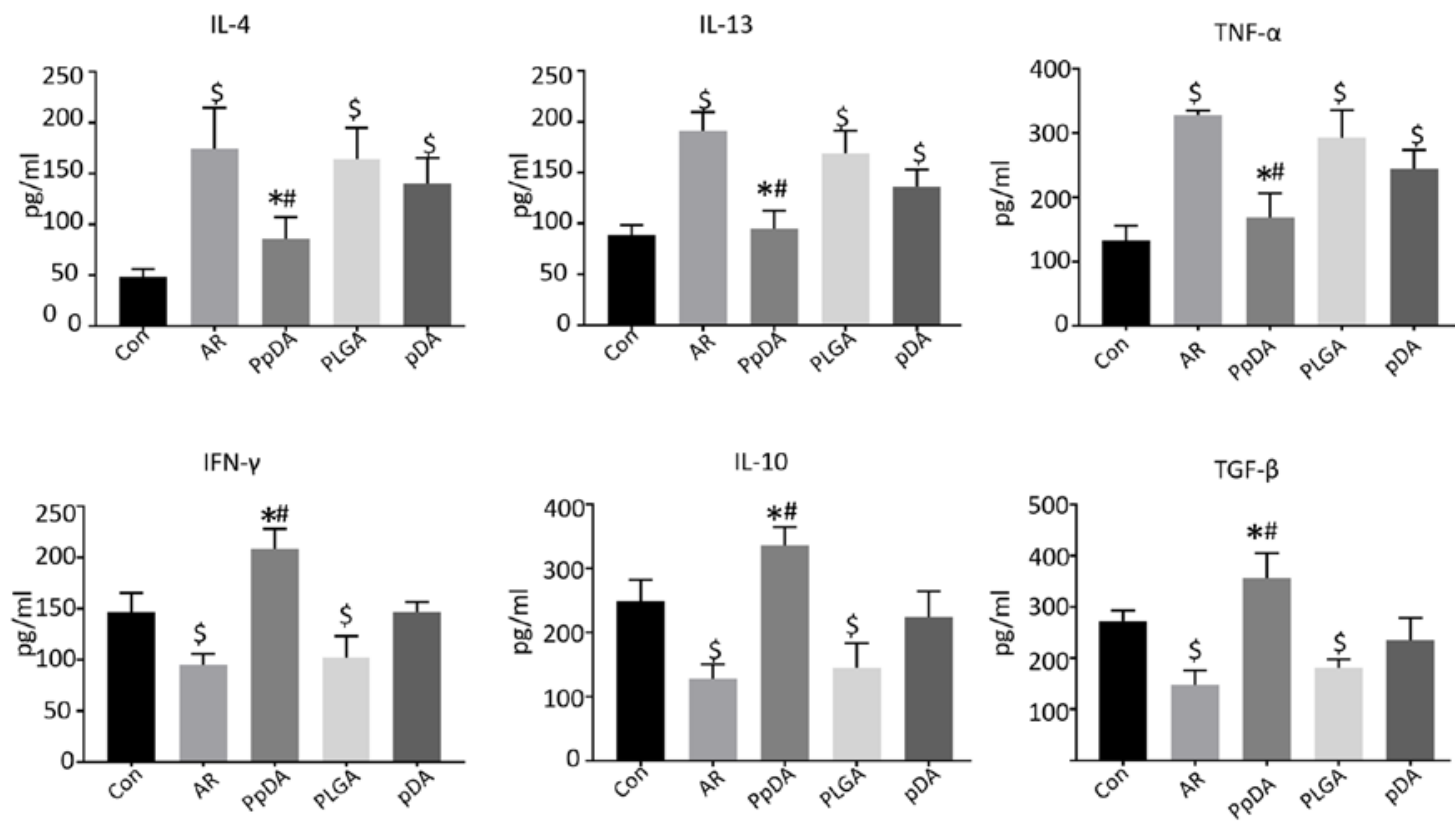

IgE
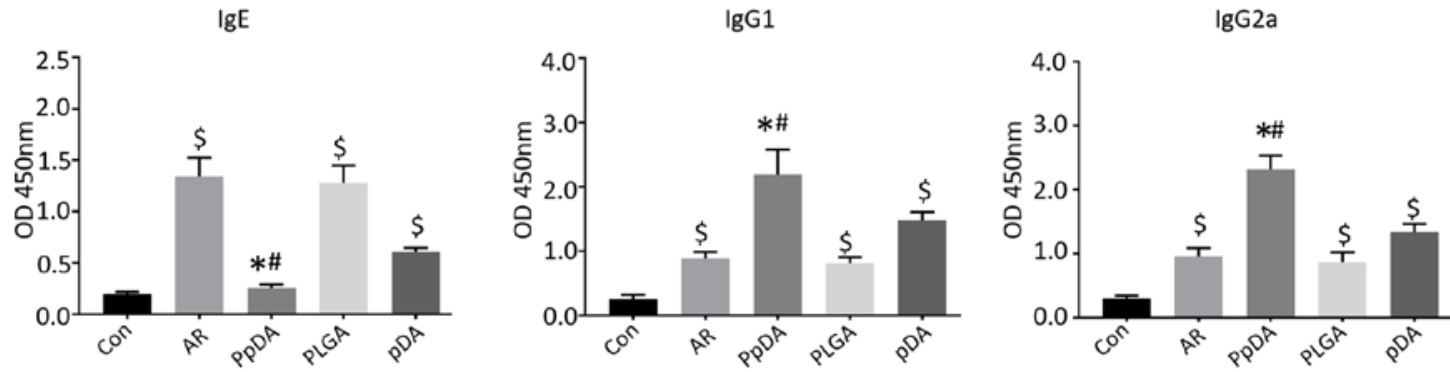

Figure 4. Evaluation of serum levels of cytokines and antibodies. Mouse sera from each group were collected and analysed by ELISA. Histogram indicates cytokine and antibody levels in each group (groups were annotated under the histogram). Data are obtained from three independent experiments and are presented as the mean \pm SD. ${ }^{\$} \mathrm{P}<0.05$ vs. the Con group; ${ }^{~} \mathrm{P}<0.01$ vs. the AR group; ${ }^{\#} \mathrm{P}<0.05$ vs. the pDA group. Con, control; AR, allergic rhinitis; PLGA, poly(L-lactide-co-glycolide).

transportation across airway epithelial cells (24). Consistent with findings of other studies, the present results revealed that intranasal administration of the DNA vaccine co-expressing an antigen and a regulatory molecular A20 ameliorated Der p2-induced nasal mucosal allergic inflammation. $N F-\kappa B$ is a key transcription factor for Th2 cell differentiation, and 
A

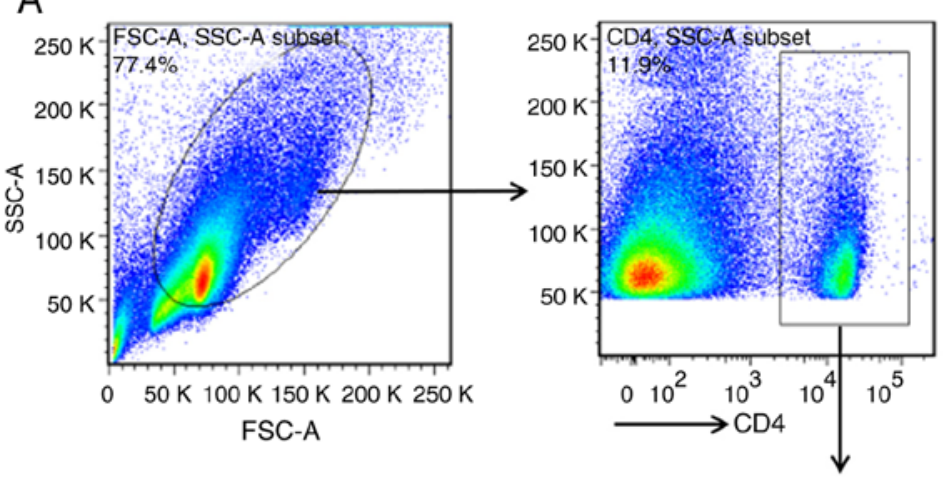

$\mathrm{B}$

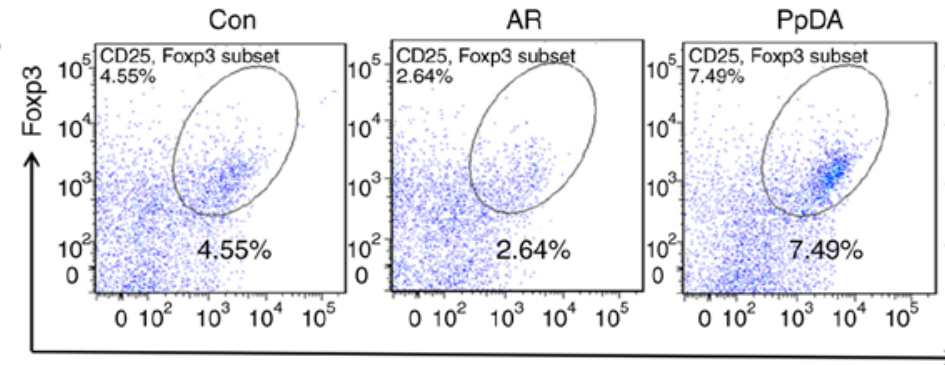

C

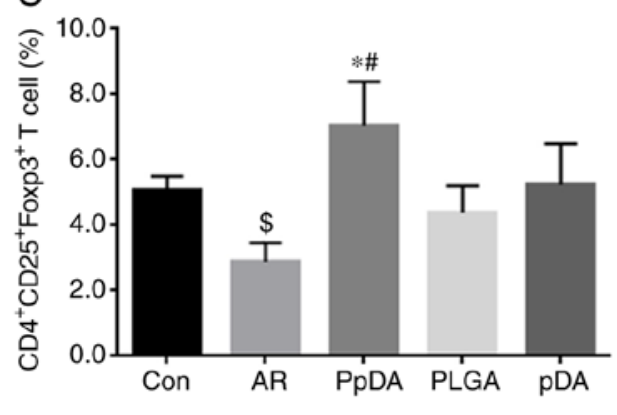

Figure 5. Splenic Treg population analysis. Mouse splenic mononuclear cells from each group were stained with fluorescently labelled anti-CD4, anti-CD25 and anti-Foxp3 antibodies and then analysed by flow cytometry. (A) Gated cells indicate CD4+T cell frequency. (B) CD4 ${ }^{+} \mathrm{T}$ cells in each group were further analyzed for $\mathrm{CD} 25^{+} \mathrm{Foxp}^{+} \mathrm{T}$ cell frequency (groups are annotated above the dot plots). (C) Bars indicate the summarized frequencies of B. Data are obtained from three independent experiments and are presented as the mean $\pm \mathrm{SD}$. ${ }^{\$} \mathrm{P}<0.05$ vs. the Con group; ${ }^{*} \mathrm{P}<0.01$ vs. the $\mathrm{AR}$ group; ${ }^{\#} \mathrm{P}<0.05$ vs. the $\mathrm{pDA}$ group. Con, control; AR, allergic rhinitis; PLGA, poly(L-lactide-co-glycolide).

its continuous activation is involved in the occurrence and development of airway allergic diseases (25). Furthermore, in our experiments, DNA vaccine treatment suppressed serum Der p2-specific IgE secretion and Th2-type cytokine (IL-4 and IL-13) expression. Neutralizing IgG subclass antibodies can inhibit IgE-mediated inflammatory responses by competing with $\operatorname{IgE}(26)$. IgG1 constitutes approximately $65 \%$ of total $\mathrm{IgG}$, and $\mathrm{IgG} 2$ constitutes approximately $22 \%$ of total $\mathrm{IgG}$. However, the contribution of allergen-specific IgG to the development of allergic inflammation is controversial (27). Data from murine experiments revealed that Th1 cells promoted IgG1 and IgG2a production by secreting IFN- $\gamma$ and that Th 2 cells promoted IgG4 production (26). Allergen immunotherapy increased protective IgG1 and IgA antibodies $(27,28)$. The results of the present study revealed that IgG1 and IgG2a production induced by DNA vaccine intranasal immunization may play a protective role in nasal allergic inflammation. Eosinophilia and mononuclear cell infiltration in the nasal mucosa are important in characterizing allergic rhinitis. The limitation of the present study is that we did not analyze eosinophilia since the amount of available nasal mucosa was not sufficient.

Regulatory $\mathrm{T}$ cells (Tregs) are negative regulatory cells of the immune response and are involved in the regulation of a variety of immune responses, including allergy, autoimmunity and graft-versus-host response (29). A previous study revealed that peripheral blood $\mathrm{CD} 4{ }^{+} \mathrm{CD} 25^{\text {high }}$ Foxp $3^{+}$Tregs were decreased in patients with mild asthma compared with those in non-asthmatic controls (30). Animal experiments have revealed that removing Tregs from mice before sensitization aggravates airway inflammation and airway hyperresponsiveness
(AHR) (31). Tregs inhibit effector T cells and regulate allergic diseases by secreting IL-10 and TGF- $\beta$, leading to reduced production of Th2 cytokines (IL-4, IL-5 and IL-13) (32). An approach to enhance the anti-allergic efficacy of therapeutic DNA vaccines was performed by fusion of a modulating cytokine such as GM-CSF, IFN- $\gamma$, IL- $1 \beta$ or an immunosuppressive molecule to the cDNA of a certain allergen (10), thus driving the immune response toward a Th1 direction. Intranasal immunization of DNA vaccine co-expressing Der p1 and ubiquitin elicited a Th1 type response, lower level of specific IgE and increased IgG in an allergic rhinitis mouse model (14). In the present study, fusion of $\mathrm{NF}-\kappa \mathrm{B}$ signaling negative regulator (A20) with Der p2 not only induced a Th1 response, but also significantly increased serum levels of IL-10 and TGF- $\beta$. In addition, the $\mathrm{CD} 4^{+} \mathrm{CD} 25^{+}$Foxp $3{ }^{+}$Treg population in the spleen was also significantly increased after Der p2-A20 DNA vaccine treatment, indicating that the DNA vaccine may inhibit the inflammatory response of AR by promoting Tregs. In the present experiment, the splenic Treg population was evaluated in each group according to published studies, because we could not collect enough cells from the nasal mucosa for flow cytometric analysis.

In conclusion, allergic rhinitis is a common disease that requires more convenient, safe and effective antigen-specific immunotherapies. A DNA vaccine co-expressing Der p2 and A20 was successfully constructed. Intranasal administration of this DNA vaccine greatly ameliorated Der p2-induced nasal mucosal proinflammatory cell infiltration by inhibiting specific IgE, IL-4 and IL-13 secretion and by increasing IgG1, IgG $2 \mathrm{a}$, IFN- $\gamma$, IL-10 and TGF- $\beta$ expression as well as the $\mathrm{CD} 4^{+} \mathrm{CD} 25^{+} \mathrm{Foxp}^{+}$Treg population. 


\section{Acknowledgements}

Not applicable.

\section{Funding}

The present study was supported by the grants from the Natural Science Foundation of China (grant nos. 81700888, $81773978,81870706)$ and the Innovation of Science and Technology Commission of Shenzhen Municipality (grant nos. JCYJ20160429091935720, JCYJ20170302165727389, JCYJ20170412103841386 and ZDSYS201506050935272).

\section{Availability of data and materials}

The datasets used and/or analyzed during the current study are available from the corresponding author on reasonable request.

\section{Authors' contributions}

WH, LM, TH, XZ, JL, BC and GY performed experiments and analyzed the data. $\mathrm{HZ}$ collected the data and revised the manuscript. ZL organized the study, supervised the experiments, designed the study and wrote the manuscript. All authors read and approved the manuscript and agree to be accountable for all aspects of the research in ensuring that the accuracy or integrity of any part of the work are appropriately investigated and resolved.

\section{Ethics approval and consent to participate}

The experiments of the present study were approved by the local Ethics Committee and comply with the current Chinese laws and are in accordance with the Declaration of Shenzhen. The experimental procedures were conducted in accordance with the approval and guidance of the Institutional Animal Care Use Committee of the ENT Institute of Shenzhen.

\section{Patient consent for publication}

Not applicable.

\section{Competing interests}

The authors declare that they have no competing interests.

\section{References}

1. Small P, Keith PK and Kim H: Allergic rhinitis. Allergy Asthma Clin Immunol 14: 51, 2018.

2. Zhang Y and Zhang L: Increasing prevalence of allergic rhinitis in china. Allergy Asthma Immunol Res 11: 156-169, 2019.

3. Hoffmann HJ, Valovirta E, Pfaar O, Moingeon P, Schmid JM Skaarup SH, Cardell LO, Simonsen K, Larché M, Durham SR and Sørensen P: Novel approaches and perspectives in allergen immunotherapy. Allergy 72: 1022-1034, 2017.

4. Jutel M, Kosowska A and Smolinska S: Allergen immunotherapy: Past, present, and future. Allergy Asthma Immunol Res 8: 191-197, 2016.

5. Cui Y, Wang Q and Jia H: Consideration of methods for identifying mite allergens. Clin Transl Allergy 8: 14, 2018.

6. Miller JD: The role of dust mites in allergy. Clin Rev Allergy Immunol Jun 23, 2018 (Epub ahead of print).
7. Reihill JA, Malcomson B, Bertelsen A, Cheung S, Czerwiec A, Barsden R, Elborn JS, Dürkop H, Hirsch B, Ennis M, et al: Induction of the inflammatory regulator A20 by gibberellic acid in airway epithelial cells. Br J Pharmacol 173: 778-789, 2016.

8. Schuijs MJ, Willart MA, Vergote K, Gras D, Deswarte K, Ege MJ Madeira FB, Beyaert R, van Loo G, Bracher F, et al: Farm dust and endotoxin protect against allergy through A20 induction in lung epithelial cells. Science 349: 1106-1110, 2015.

9. Tulic MK: Allergen-free immunotherapy using DNA vaccines in treatment of established allergic disease. Clin Exp Allergy 42: 3-4, 2012.

10. Weiss R, Hammerl P, Hartl A, Hochreiter R, Leitner WW, Scheiblhofer $S$ and Thalhamer J: Design of protective and therapeutic DNA vaccines for the treatment of allergic diseases. Curr Drug Targets Inflamm Allergy 4: 585-597, 2005.

11. Fonseca DM, Wowk PF, Paula MO, Campos LW, Gembre AF, Turato WM, Ramos SG, Dias-Baruffi M, Barboza R, Gomes E, et al: Recombinant DNA immunotherapy ameliorate established airway allergy in a IL-10 dependent pathway. Clin Exp Allergy 42: 131-143, 2012.

12. Hu T, Fan X, Ma L, Liu J, Chang Y, Yang P, Qiu S, Chen T, Yang L and Liu Z: TIM4-TIM1 interaction modulates Th2 pattern inflammation through enhancing SIRT1 expression. Int J Mol Med 40: 1504-1510, 2017.

13. Wikstrom ME, Batanero E, Smith M, Thomas JA, von Garnier C Holt PG and Stumbles PA: Influence of mucosal adjuvants on antigen pass age and CD4+ $\mathrm{T}$ cell activation during the primary response to airborne allergen. J Immunol 177: 913-924, 2006.

14. Ou J, Shi W, Xu Y and Tao Z: Intranasal immunization with DNA vaccine coexpressing Der $\mathrm{p} 1$ and ubiquitin in an allergic rhinitis mouse model. Ann Allergy Asthma Immunol 113: 658-665.e651, 2014.

15. ChenZ, TaoZZ,Zhou XH, Wu TT and Ye LF: Immunosuppressive effect of sinomenine in an allergic rhinitis mouse model. Exp Ther Med 13: 2405-2410, 2017.

16. Rothenberg ME, Saito $\mathrm{H}$ and Peebles RS Jr: Advances in mechanisms of allergic disease in 2016. J Allergy Clin Immunol 140: $1622-1631,2017$

17. Gholami E, Oliveira F, Taheri T, Seyed N, Gharibzadeh S, Gholami N, Mizbani A, Zali F, Habibzadeh S, Bakhadj DO, et al: DNA plasmid coding for Phlebotomus sergenti salivary protein PsSP9, a member of the SP15 family of proteins, protects against Leishmania tropica. PLoS Negl Trop Dis 13: e0007067, 2019.

18. Asbach B, Kibler KV, Kostler J, Perdiguero B, Yates NL, Stanfield-Oakley S, Tomaras GD, Kao SF, Foulds KE, Roederer M, et al: Priming with a potent HIV-1 DNA vaccine frames the quality of immune responses prior to a poxvirus and protein boost. J Virol 93: e01529, 2019.

19. Dantzer JA and Wood RA: Next-generation approaches for the treatment of food allergy. Curr Allergy Asthma Rep 19: 5, 2019.

20. Scheiblhofer S, Thalhamer J and Weiss R: DNA and mRNA vaccination against allergies. Pediatr Allergy Immunol 29: 679-688, 2018.

21. Su Y, Connolly M, Marketon A and Heiland T: CryJ-LAMPDNA vaccines for Japanese red cedar allergy induce robust Th1-Type immune responses in murine model. J Immunol Res 2016: 4857869, 2016.

22. Pulsawat P, Pitakpolrat P, Prompetchara E, Kaewamatawong T, Techakriengkrai N, Sirivichayakul S, Buranapraditkun S, Hannaman D, Ruxrungtham K and Jacquet A: Optimization of a Der $\mathrm{p}$ 2-based prophylactic DNA vaccine against house dust mite allergy. Immunol Lett 151: 23-30, 2013.

23. Das T, Chen Z, Hendriks RW and Kool M: A20/tumor necrosis factor $\alpha$-induced protein 3 in immune cells controls development of autoinflammation and autoimmunity: Lessons from mouse models. Front Immunol 9: 104, 2018.

24. Li TL, Zhang SY, Du YC and Yang PC: Deficiency of ubiquitin A20 promotes antigen transport across airway epithelial cells via a transcellular pathway. Anal Biochem 433: 86-91, 2013.

25. Kelly C, Shields MD, Elborn JS and Schock BC: A20 regulation of nuclear factor- $\kappa \mathrm{B}$ : Perspectives for inflammatory lung disease. Am J Respir Cell Mol Biol 44: 743-748, 2011.

26. Scott-Taylor TH, Axinia SC, Amin S and Pettengell R: Immunoglobulin $\mathrm{G}$; structure and functional implications of different subclass modifications in initiation and resolution of allergy. Immun Inflamm Dis 6: 13-33, 2018.

27. Williams JW, Tjota MY and Sperling AI: The contribution of allergen-specific IgG to the development of th2-mediated airway inflammation. J Allergy (Cairo) 2012: 236075, 2012. 
28. Wisniewski J, Agrawal R and Woodfolk JA: Mechanisms of tolerance induction in allergic disease: Integrating current and emerging concepts. Clin Exp Allergy 43: 164-176, 2013.

29. Maloy KJ and Powrie F: Regulatory T cells in the control of immune pathology. Nat Immunol 2: 816-822, 2001.

30. Baatjes AJ, Smith SG, Watson R, Howie K, Murphy D, Larché M, Denburg JA, Inman MD and O'Byrne PM: T regulatory cell phenotypes in peripheral blood and bronchoalveolar lavage from non-asthmatic and asthmatic subjects. Clin Exp Allergy 45: 1654-1662, 2015.

31. Lewkowich IP, Herman NS, Schleifer KW, Dance MP, Chen BL, Dienger KM, Sproles AA, Shah JS, Köhl J, Belkaid Y and Wills-Karp M: $\mathrm{CD} 4{ }^{+} \mathrm{CD} 25^{+} \mathrm{T}$ cells protect against experimentally induced asthma and alter pulmonary dendritic cell phenotype and function. J Exp Med 202: 1549-1561, 2005.
32. Chaisri U, Tungtrongchitr A, Indrawattana N, Meechan $P$, Phurttikul W, Tasaniyananda N, Saelim N, Chaicumpa W and Sookrung N: Immunotherapeutic efficacy of liposome-encapsulated refined allergen vaccines against Dermatophagoides pteronyssinus allergy. PLoS One 12: e0188627, 2017.

(7)(3) This work is licensed under a Creative Commons Attribution-NonCommercial-NoDerivatives 4.0 International (CC BY-NC-ND 4.0) License. 